\title{
Bedaquiline as a potential agent in the treatment of Mycobacterium abscessus infections
}

To the Editor:

Mycobacterium abscessus is increasingly being recognised as a significant human pathogen, especially in patients with cystic fibrosis, and the specific $M$. abscessus subspecies seems to influence the clinical outcome [1]. The pulmonary manifestation of this nontuberculous mycobacteria (NTM) infection is one of the most difficult to treat forms, leading to substantial morbidity and mortality in this population $[1,2]$. M. abscessus strains are highly resistant to most antibacterial drugs [2]. The recent development of liposomal amikacin for inhalation for patients with cystic fibrosis suggested a therapeutic breakthrough. However, even though sputum conversion was improved in a phase 2 study, the primary end-point (change from baseline to day 84 on a semi-quantitative mycobacterial growth scale) was not reached [3]. We analysed the minimal inhibitory concentration (MIC) of another promising new anti-tuberculous drug, bedaquiline, using 20 clinical isolates of $M$. abscessus. No systematic studies on the distribution of MIC for M. abscessus have been performed previously.

Bedaquiline is a diarylquinoline drug recently licensed for the treatment of multidrug-resistant Mycobacterium tuberculosis infections. It acts through inhibition of the mycobacterial $\mathrm{F}_{1} \mathrm{~F}_{0}$ - ATP synthase, [4] and is characterised by excellent intracellular bactericidal activity and a high accumulation rate [4]. In previous studies, bedaquiline showed excellent in vitro activity against $M$. tuberculosis, including multidrug-resistant strains [5]. The new drug has been successfully and safely used in the treatment of both adult and paediatric multidrug-resistant and extensively drug-resistant tuberculosis alike [6,7], even over extended periods of as long as 18 months [8].

As for other NTM, in vitro drug susceptibility testing of $M$. abscessus strains using conventional drugs is recommended only after treatment failure occurs [2]. We present the in vitro bedaquiline MIC results of 20 clinical strains of $M$. abscessus isolated in our centre between 2011 and 2016 from patients with pulmonary NTM disease, including three patients with cystic fibrosis. In this study, 4/20 strains (20\%) were classified as $M$. abscessus subspecies bolletii, while all others belonged to the M. abscessus subspecies abscessus. The thirdM. abscessus subspecies, M. abscessus subspecies massiliense was not identified among our strains. MIC was determined by a modified agar dilution method on Middlebrook $7 \mathrm{H} 10$ agar, as described previously [9]. MIC was defined as the lowest drug concentration that inhibited at least $99 \%$ of the bacterial proportion after a two-fold serial dilution of the respective drug (MIC99).

Results are shown in table 1. All M. abscessus strains tested exhibited a MIC for bedaquiline of $\leqslant 1 \mu \mathrm{g} \cdot \mathrm{mL}^{-1}$, and $17 / 20$ (85\%) had a MIC of $\leqslant 0.5 \mu \mathrm{g} \cdot \mathrm{mL}^{-1}$. Median MIC for all M. abscessus strains was $0.5 \mu \mathrm{g} \cdot \mathrm{mL}^{-1}$, only slightly higher than that for M. tuberculosis $\left(0.4 \mu \mathrm{g} \cdot \mathrm{mL}^{-1}\right)$ [10]. For each of the three M. abscessus strains isolated from patients with cystic fibrosis, the MIC was also $0.5 \mu \mathrm{g} \cdot \mathrm{mL}^{-1}$. M. abscessus subspecies bolletii showed a trend towards lower MIC values than M. abscessus subspecies abscessus (chi-squared test: $\mathrm{p}=0.135$ ). To our knowledge, this is the first series of published MIC values for clinical M. abscessus strains tested against bedaquiline. The single strains tested by OBREGón-HeNaO et al. [11] and ANDRIES et al. [12] revealed MICs of $1.0 \mu \mathrm{g} \cdot \mathrm{mL}^{-1}$ and $0.25 \mu \mathrm{g} \cdot \mathrm{mL}^{-1}$, respectively, both similar to ours. The European Committee on Antimicrobial Susceptibility Testing break-point for $M$. tuberculosis is $0.25 \mu \mathrm{g} \cdot \mathrm{mL}^{-1}$, thus $3 / 20$ strains $(15 \%)$ had a MIC equal to or lower than this break-point, and most strains $(17 / 20,85 \%)$ showed a MIC below or only slightly higher than that $\left(\leqslant 0.5 \mu \mathrm{g} \cdot \mathrm{mL}^{-1}\right)$.

@ERSpublications

Bedaquiline may be a potential agent to treat severe or relapsing Mycobacterium abscessus infection http://ow.ly/3lUp309VZVj

Cite this article as: Vesenbeckh S, Schönfeld N, Roth A, et al. Bedaquiline as a potential agent in the treatment of Mycobacterium abscessus infections. Eur Respir J 2017; 49: 1700083 [https://doi.org/10.1183/ 13993003.00083-2017]. 
TABLE 1 Minimal inhibitory concentrations (MIC) of bedaquiline for 20 Mycobacterium abscessus strains, according to subspecies (chi-squared test: $\mathrm{p}=0.135$ )

\begin{tabular}{lccccc} 
M. abcessus subspecies & Strains $\mathbf{n}$ & \multicolumn{5}{c}{${\text { MIC } \boldsymbol{\mu g} \cdot \mathbf{m L}^{-1}}^{-1}$} \\
\cline { 3 - 6 } & & $\mathbf{0 . 1 2}$ & $\mathbf{0 . 2 5}$ & $\mathbf{0 . 5}$ & $\mathbf{1 . 0}$ \\
\hline M. abcessus subsp. bolletii & 4 & $1 / 20(5 \%)$ & $1 / 20(5 \%)$ & $2 / 20(10 \%)$ & 0 \\
M. abcessus subsp. abscessus & 16 & 0 & $1 / 20(5 \%)$ & $12 / 20(60 \%)$ & $3 / 20(15 \%)$ \\
\hline
\end{tabular}

Keeping in mind that only a few treatment options for M. abscessus infections are available [1, 2], bedaquiline could be an effective alternative in the multidrug second-line therapy of this mycobacterial disease. At standard oral doses, bedaquiline maintained a mean plasma concentration of $0.6 \mathrm{mg} \cdot \mathrm{L}^{-1}$, as shown in early pharmacokinetic studies [13], and it was extensively distributed to tissues, including the lungs [12]. Bedaquiline significantly reduced the bacterial burden in the lungs after 4 days of treatment at least in one M. abscessus -infected mouse model [11], but by contrast, LERAT et al. [14] reported in 2014 that bedaquiline showed almost no activity in his nude mice. Philley et al. [15] reported that when bedaquiline was used as salvage therapy in a human study of four patients with M. abscessus disease, there was clinical improvement in all cases at 3 months of treatment, a sustained reduction of bacterial load in sputum in half of all patients, and no severe side effects. However, after 6 months of observation, only one patient still showed improvement of clinical symptoms. In the study reported by PHILLEY et al. [15], the same bedaquiline doses as those recommended for $M$. tuberculosis were used. Owing to the high intracellular accumulation rate of the lipophilic drug bedaquiline, it is likely that even with a slightly higher MIC compared with that for M. tuberculosis, the drug will be effective against M. abscessus strains without increasing drug doses. Early bactericidal activity trials might be helpful in selectively demonstrating the in vivo activity of bedaquiline without other background combination drugs.

Considering the rising incidence of $M$. abscessus infections worldwide as well as the frequent multidrug resistance with subsequent treatment failure, bedaquiline could be an alternative in multidrug treatment regimens for severe or relapsing disease, potentially including patients with underlying cystic fibrosis. In our study, only three patients with underlying cystic fibrosis were included, thus clinical trials involving patients with cystic fibrosis are necessary to confirm this potential use.

Silvan Vesenbeckh ${ }^{1}$, Nicolas Schönfeld ${ }^{1}$, Andreas Roth ${ }^{2}$, Gudrun Bettermann ${ }^{3}$, David Krieger ${ }^{1}$, Torsten Thomas Bauer ${ }^{1,4}$, Holger Rüssmann ${ }^{3}$ and Harald Mauch ${ }^{3}$

${ }^{1}$ Klinik für Pneumologie, Lungenklinik Heckeshorn, HELIOS Klinikum Emil von Behring, Berlin, Germany. ${ }^{2}$ Institut für Gewebediagnostik, HELIOS Klinikum Emil von Behring, Berlin, Germany. ${ }^{3}$ Institut für Mikrobiologie, Immunologie und Laboratoriumsmedizin, HELIOS Klinikum Emil von Behring, Berlin, Germany. ${ }^{4}$ Deutsches Zentralkomitee zur Bekämpfung der Tuberkulose (DZK), Berlin, Germany.

Correspondence: Silvan Vesenbeckh, Klinik für Pneumologie, Lungenklinik Heckeshorn, HELIOS Klinikum Emil von Behring, Walterhoeferstr. 11, 14165 Berlin, Germany. E-mail: silvan.vesenbeckh@helios-kliniken.de

Received: Jan 132017 | Accepted after revision: Feb 132017

Conflict of interest: None declared.

\section{References}

1 Robinson PD, Harris KA, Aurora P, et al. Paediatric lung transplant outcomes vary with Mycobacterium abscessus complex species. Eur Respir J 2013; 41: 1230-1232.

2 Schönfeld N, Haas W, Richter E, et al. Recommendations of the German Central Committee against Tuberculosis (DZK) and the German Respiratory Society (DGP) for the diagnosis and treatment of non-tuberculous mycobacterioses. Pneumologie 2016; 70: 250-276.

3 Olivier KN, Griffith DE, Eagle G, et al. Randomized trial of liposomal amikacin for inhalation in nontuberculous mycobacterial lung disease. Am J Respir Crit Care Med 2017; 195: 814-823.

4 Hards K, Robson JR, Berney M, et al. Bactericidal mode of action of bedaquiline. J Antimicrob Chemother 2015; 70: 2028-2037.

5 Diacon AH, Pym A, Grobusch MP, et al. Multidrug-resistant tuberculosis and culture conversion with bedaquiline. N Engl J Med 2014; 371: 723-732.

6 Pontali E, Sotgiu G, D'Ambrosio L, et al. Bedaquiline and multidrug-resistant tuberculosis: a systematic and critical analysis of the evidence. Eur Respir J 2016; 47: 394-402.

7 Tadolini M, Garcia-Prats AJ, D’Ambrosio L, et al. Compassionate use of new drugs in children and adolescents with multidrug-resistant and extensively drug-resistant tuberculosis: early experiences and challenges. Eur Respir J 2016; 48: 938-943.

8 Lewis JM, Hine P, Walker J, et al. First experience of effectiveness and safety of bedaquiline for 18 months within an optimised regimen for XDR-TB. Eur Respir J 2016; 47: 1581-1584. 
9 Schönfeld N, Bergmann T, Vesenbeckh S, et al. Minimal inhibitory concentrations of first-line drugs of multidrug-resistant tuberculosis isolates. Lung India 2012; 29: 309-312.

10 Keller PM, Homke R, Ritter C, et al. Determination of MIC distribution and epidemiological cutoff values for bedaquiline and delamanid in Mycobacterium tuberculosis using the MGIT 960 system equipped with TB eXiST. Antimicrob Agents Chemother 2015; 59: 4352-4355.

11 Obregón-Henao A, Arnett KA, Henao-Tamayo M, et al. Susceptibility of Mycobacterium abscessus to antimycobacterial drugs in preclinical models. Antimicrob Agents Chemother 2015; 59: 6904-6912.

12 Andries K, Verhasselt P, Guillemont J, et al. A diarylquinoline drug active on the ATP synthase of Mycobacterium tuberculosis. Science 2005; 307: 223-227.

13 Diacon AH, Pym A, Grobusch M, et al. The diarylquinoline TMC207 for multidrug-resistant tuberculosis. $N$ Engl J Med 2009; 360: 2397-2405.

14 Lerat I, Cambau E, Roth Dit Bettoni R, et al. In vivo evaluation of antibiotic activity against Mycobacterium abscessus. J Infect Dis 2014; 209: 905-912.

15 Philley JV, Wallace RJ Jr, Benwill JL, et al. Preliminary results of bedaquiline as salvage therapy for patients with nontuberculous mycobacterial lung disease. Chest 2015; 148: 499-506. 